\title{
A study comparing endogenous protoporphyrin IX induced by 5-ALA and ALA-methyl ester with exogenous PpIX and PpIX dimethyl ester in photodynamic diagnosis of human nasopharyngeal carcinoma xenografts
}

\author{
VANAJA MANIVASAGER ${ }^{1}$, KAREN KAR LYE YEE ${ }^{1}$, PAUL WAN SIA HENG ${ }^{2}$, \\ KHEE CHEE SOO ${ }^{3}$ and MALINI OLIVO ${ }^{1}$ \\ ${ }^{1}$ Division of Medical Sciences, National Cancer Centre, 11 Hospital Drive, Singapore 169610; \\ ${ }^{2}$ Department of Pharmacy, National University of Singapore, 18 Science Drive 4, Singapore 117543, Singapore \\ Received February 17, 2006; Accepted April 19, 2006
}

\begin{abstract}
Aminolevulinic acid (5-ALA) and its esters have been under intense investigation to enhance the endogenous production of protoporphyrin IX (PpIX) in tumour cells for the purpose of photodynamic diagnosis. In this study we have investigated the use of exogenous PpIX and its dimethyl ester (PME) and compared the results with endogenous PpIX produced via 5-ALA and ALA methyl ester (AME) in poorly differentiated NPC/CNE-2 nasopharyngeal carcinoma cells in both in vivo and in vitro systems. All prodrugs and photosensitizers were administered to tumour bearing balb/c nude mice either intravenously or topically. In vitro results show that 5-ALA induced more PpIX fluorescence when compared with AME in NPC/CNE-2 cells and PME showed better uptake than PpIX. In vivo results show that exogenous PpIX and PME show promise as good candidates as photosensitizers for photodynamic diagnosis as they exhibit significant selectivity between tumour tissue and normal tissue at $3 \mathrm{~h}$. Modification of delivery vehicle used for application of exogenous PpIX and PME could allow for rapid uptake; better selectivity and localization of the photosensitizer.
\end{abstract}

\section{Introduction}

Fluorescence diagnosis is a promising new method for the detection and treatment of malignant and pre-malignant lesions. The use of porphyrins as a fluorescence marker to diagnose superficial and accessible cancers non-invasively is being extensively studied and developed (1-4). Porphyrins are photosensitive and fluoresce when exposed to illumination with

Correspondence to: Dr Malini Olivo, Division of Medical Sciences, National Cancer Centre, 11 Hospital Drive, Singapore 169610 E-mail:dmsmcd@nccs.com.sg

Key words: protoporphyrin IX, protoporphyrin IX dimethyl ester, 5-aminolevulinic acid, 5-aminolevulinic acid methyl ester, photodynamic diagnosis light of specific wavelength. Exogenously applied sensitizers accumulate selectively in malignant lesions and induce fluorescence. Hematoporphyrin Derivative $(\mathrm{HpD})$ and Porfimer Sodium (Photofrin ${ }^{\circledR}$ ) were the first and most widely studied and used photosensitizers in patients for photodynamic diagnosis and therapy (5). HpD contains a complex mixture of porphyrin components. However, not all of them are photodynamically active in vivo. Photofrin is a purified version of $\mathrm{HpD}$. In both cases, one of the active components is Protoporphyrin IX (PpIX) (6-8). In 1955, Scott (9) described the transitory hypersensitivity to sunlight following exogenous administration of 5-aminolevulinic acid (5-ALA), which induced endogenous PpIX. 5-ALA is a naturally occurring precursor in the heme biosynthetic pathway of nucleated cells (10-12). Heme biosynthesis consists of eight discrete enzyme-catalysed steps that involve the mitochondrial and cytosolic compartments of the cell. 5-ALA by itself is not a photosensitive component. It is produced in the mitochondria and actively transported into the cytoplasm where the intermediate steps of heme biosynthesis pathway take place following its re-entry into mitochondria. Here, PpIX is produced. This is the last step before forming heme by insertion of ferrous iron by the enzyme ferrochelatase (FC). FC chelates the $\mathrm{Fe}^{2+}$ into the porphyrin ring structure. A tight feedback loop control ensures that only a very small amount of PpIX is left at any one time. Accumulation of excess endogenous porphyrins induced by excess 5-ALA only happens under abnormal conditions such as in malignant or pre-malignant cell transformation. In such conditions, the activity of two main enzymes porphobilinogen deaminase (PBG) and FC are changed (13-15). In abnormal cells, while the activity of PBG is increased, the activity of FC is decreased resulting in the build up of PpIX. In normal cells, FC catalyses the conversion of photosensitive PpIX to heme, which is not photosensitive. The over-production of PpIX can also be induced by the excess administration of exogenous 5-ALA or ALA esters (topically or intravenously).

The hydrophilic properties of 5-ALA limit its uptake and penetration into tissue, particularly after topical application. However, in vitro studies have demonstrated that esterification of 5-ALA increases its lipophilicity, thereby improving pene- 
tration through cellular membranes and circumvents the limitations. After penetration the ester derivative has to be hydrolyzed to free 5-ALA by non-specific esterases to produce high PpIX fluorescence. In this study we have investigated the use of 5-ALA and its ester derivative ALA methyl ester (AME) induced PpIX in the fluorescence detection of nasopharyngeal carcinoma in vitro and in vivo. We have also investigated the use of exogenous PpIX and its dimethyl ester derivative (PME) as photosentizers and compared them to endogenous PpIX induced by 5-ALA and AME.

\section{Materials and methods}

Drugs. 5-ALA (Medac, Germany); PpIX and PME (Frontier Scientific Inc. Porphyrin Products, UT, USA) were stored in desiccators at room temperature. AME (Sigma-Aldrich) was stored in desiccators at $4^{\circ} \mathrm{C}$. PpIX stock solution $(10 \mathrm{mM})$ was made up in dimethyl sulfoxide (DMSO) and $10 \mathrm{mM}$ PME stock solution was made up in hydrochloric acid and sodium hydroxide. 5-ALA and AME were made up in $0.9 \%$ sodium chloride and cold de-ionized water respectively as and when required.

In vitro cellular uptake studies. The poorly differentiated human nasopharyngeal carcinoma cell line NPC/CNE-2 (a gift from Professor K.M. Hui, Cellular and Molecular Research, National Cancer Centre, Singapore) and normal human lung fibroblasts (NHLF) were used. The NPC/CNE-2 cells were grown in culture medium RPMI-1640 supplemented with $10 \%$ fetal bovine serum (FBS; Hyclone, Logan, UT), 1X L-glutamine, 100 units/ml penicillin and streptomycin (Gibco) and $1 \mathrm{X}$ sodium pyruvate in an atmosphere of $5 \% \mathrm{CO}_{2}$ at $37^{\circ} \mathrm{C}$. Confluent cells were trypsinized with $0.05 \%$ Trypsin-EDTA (Gibco) and seeded onto $100-\mathrm{mm}$ petri dishes $\left(1.5 \times 10^{6}\right.$ cells/dish $)$ and left to settle and attach overnight. NHLF cells were grown using fibroblast growth medium-2 (FGM-2). FGM-2 (5000 ml) contained $1 \mathrm{mg} / \mathrm{ml}$ human recombinant fibroblast growth factor; $5 \mathrm{mg} / \mathrm{ml}$ insulin; $50 \mathrm{mg} / \mathrm{ml}$ gentamycin; $50 \mathrm{mg} / \mathrm{ml}$ amphotericin; and $10 \mathrm{ml}$ of FBS. The culture medium was removed and the cells were washed twice with phosphate buffered saline (PBS). The petri dishes were divided into four experimental groups and a control group. The cells were incubated with $15 \mathrm{mM}$ ALA and AME; $2 \mu \mathrm{M}$ PpIX and PME in culture medium without serum for $30 \mathrm{~min}$. The cells were then washed with PBS. Fresh medium with serum was added to the dishes and incubated at $37^{\circ} \mathrm{C}$ with $5 \% \mathrm{CO}_{2}$ for $1,3,6,9$ and $24 \mathrm{~h}$. At each time-point the cells were washed with PBS, scraped and incubated in perchloric acid and methanol mixture (1:1) for 15 min. 5-ALA-PpIX and AME-PpIX and exogenous PpIX and PME were detected using the spectrofluorophotometer RF-5301 PC (Shimadzu RF-5301PC, $\lambda_{\mathrm{ex}}=$ $\left.400 \mathrm{~nm}, \lambda_{\mathrm{em}}=500-700 \mathrm{~nm}\right)$.

Animal experiments and tumour model. Male Balb/c nude mice, 6-8 weeks old (Animal Resource Centre, Perth, Australia) were used in a licensed biomedical research facility and all procedures were carried out in adherence to the rules and regulations of the Institutional Animal Care and Use Committee (IACUC) of National Cancer Centre of Singapore.
Cells were cultured and implanted subcutaneously $\left(1 \times 10^{6}\right)$ onto the flanks of the nude mice. The tumour was allowed to grow to a size of 5-8 $\mathrm{mm}$ in diameter. The animals were divided into groups and were given either intravenous or topical administration of 5-ALA; AME; PpIX or PME.

Intravenous administration and fluorescence imaging. The mice were administered with a dose of $250 \mathrm{mg} / \mathrm{kg}$ body weight of 5-ALA and AME by tail vein injections. As for PpIX or PME, $1 \mathrm{mg} / \mathrm{kg}$ body weight was administered intravenously to the mice. They were then kept in the darkness until the various time-points. A cocktail of Hypnorm $(0.315 \mathrm{mg} / \mathrm{ml}$ fentanyl citrate and $10 \mathrm{mg} / \mathrm{ml}$ flaunisone, Janssen), Dormicum ( $5 \mathrm{mg} / \mathrm{ml}$ midazolam $\mathrm{HCl}$, David Bull Laboratories) and deionised water was used to anaesthetize the mice. The skin overlaying the tumour was carefully removed to expose the tumour $(10,11)$. Extreme care was taken to minimize bleeding as this can interfere with the imaging procedure. The tumour and the surrounding normal tissue were imaged using the Karl Storz fluorescence endoscope system (D-Light AF system, Karl Storz, Tuttlingen, Germany). A xenon short arc lamp was used for both the white light illumination and the PpIX fluorescence excitation filtered by a band pass filter $(375-480 \mathrm{~nm})$. Both illumination and observation of tissue of interest were achieved via a modified endoscope integrated with a long pass (LP) filter (cut-off wavelength at $560 \mathrm{~nm}$ ). The fluorescence and white light images were recorded by a sensitive color CCD video camera (Tricam SL-PDD, Karl Storz, Tuttlingen, Germany) connected to the modified endoscope. A white light image of the tumour was obtained at the end of the last time-point. This is to help later in identifying the tumour and its margins when comparing with the fluorescence image.

Topical applications. The mice were anaesthetized and their skin overlaying the tumour was removed before the 5-ALA and AME $\left(2.5 \mathrm{mg} / \mathrm{cm}^{2}\right)$ topical application in the form of patches were applied making sure that the patches covered the tumour adequately and part of the normal tissue surrounding the tumour. The patches were applied for $30 \mathrm{~min}$ and then removed. The area was rinsed well with $70 \%$ alcohol to remove any excess residual drug. The xenografts and surrounding normal tissue were imaged at various time-points post patch application.

Topical application of PpIX/PME. The gels consisted of Gantrez [International Speciality Products (ISP), USA] and polyvinyl-pyrrolidone (PVP) (Plasdone, ISP, USA) polymers. Pharmasolve (ISP, USA) was used as a solvent. The gels were made using a combination of PVP and Gantrez in the proportions of 8:2 and 6:4. Another gel was constituted using Gantrez and Stabilez 06. Both polymers, PVP and Gantrez, were dissolved thoroughly in Pharmasolve. PpIX or PME was dissolved in the gel at $5 \%(\mathrm{wt} / \mathrm{vol})$ proportion and applied as evenly as possible with a brush. After 30-min incubation the remaining gel was wiped off with $70 \%$ alcohol. The tumours and surrounding normal muscle tissues were imaged at 1,3 and $6 \mathrm{~h}$ post gel application.

Image analysis. The images thus obtained were analyzed using the image analysis software, MicroImage 4.0 (Olympus 

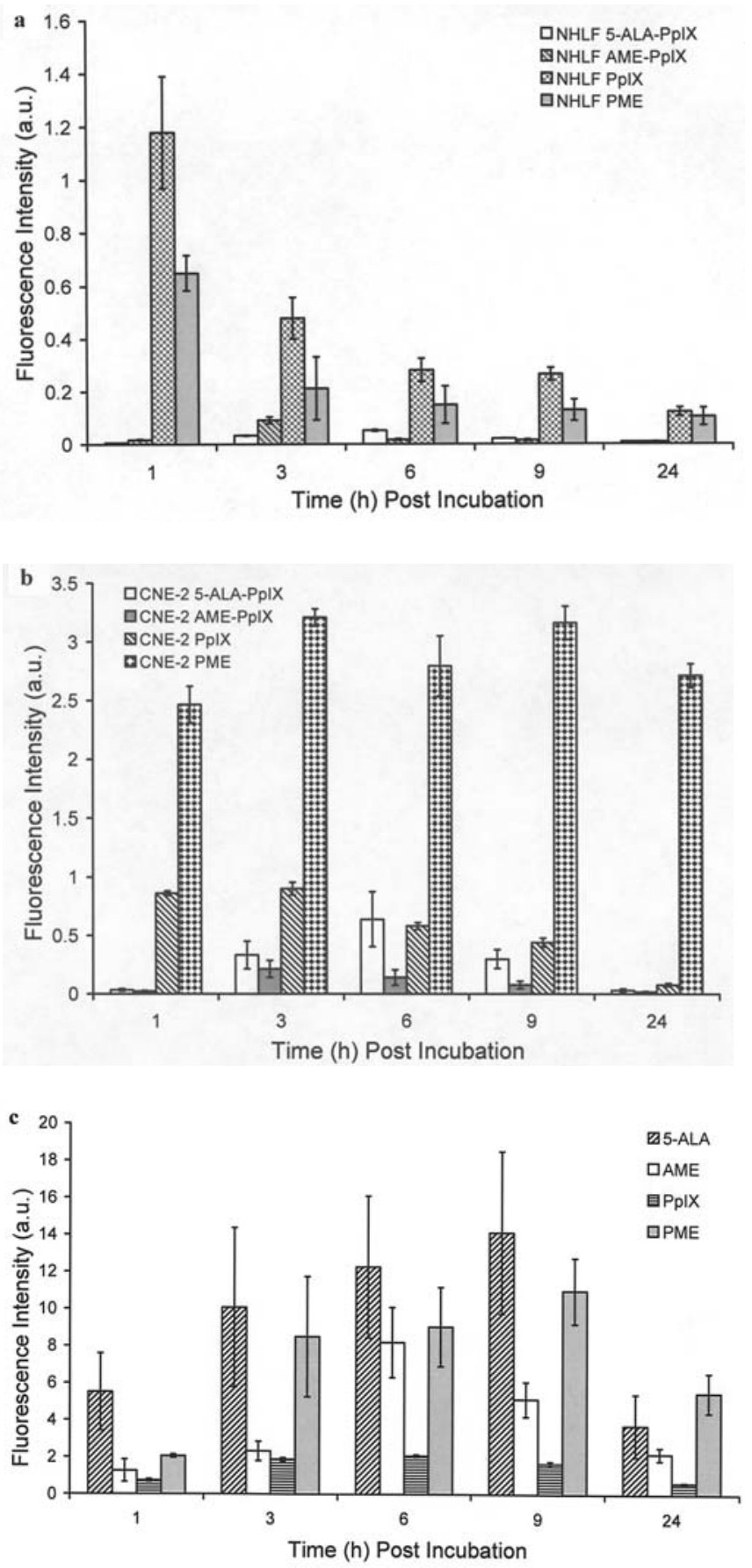

Figure 1. Fluorescence intensity of 5-ALA - PpIX; AME - PpIX; exogenous PpIX and PME in NHLF cells. b, Fluorescence intensity of 5-ALA - PpIX; AME - PpIX; exogenous PpIX and PME in NPC/CNE-2 cells. c, Ratio of fluorescence intensity of 5-ALA - PpIX; AME - PpIX; exogenous PpIX and $\mathrm{PME}$ in NPC/CNE-2 cells and NHLF cells.

Optical Co. Europa). Images obtained in blue light needed to be further processed to obtain normalized fluorescence intensity images. The processing involves contrast enhancement of the original image. This is followed by hue extraction where one extracts the fluorescence colour (red) that is needed. Following hue extraction, the thresholding and segmentation of the tumour was carried out to isolate the area of interest. This gives us the red-black image. Using the margin from this we obtain the fluorescence intensity of the tumour and normal regions by dividing the intensity of red by the intensity of blue in the same region. Finally, the normalized fluorescence intensity of image was obtained.

Statistical analysis. Statistical analysis was performed using SPSS v10.0 (SPSS Inc., Chicago, USA) for windows. Unpaired t-test was used to establish the significance of differences between groups at the same time-point. Comparisons of in vivo fluorescence between groups at different time-points were determined by One-way ANOVA. P-values $\leq 0.05$ were considered significant. Results are expressed as the mean \pm standard error of the mean (SEM) unless indicated otherwise.

\section{Results}

NPC/CNE-2 cells were compared with normal human lung fibroblasts (NHLF). Fig. 1a and b shows the fluorescence intensity of 5-ALA and AME induced PpIX, (5-ALA-PpIX and AME-PpIX) exogenous PpIX and PME in the two cells lines. In the case of the normal cell line, while 5-ALA-PpIX and AME-PpIX exhibited low fluorescence intensity, exogenous PpIX and PME exhibited high fluorescence intensity at the initial time-point. On the other hand, NPC/CNE-2 cells exhibited high uptake of PME from initial time-point. Fluorescence intensity of exogenous PpIX and 5-ALA-PpIX were comparable between 6 and $9 \mathrm{~h}$ post incubation, whereas AME-PpIX exhibited low fluorescence intensity throughout. Fig. 1c shows the ratio of tumour cells to normal cells. From as early as $1 \mathrm{~h}$, the tumour to normal ratio ( $\mathrm{T} / \mathrm{N}$ ratio) of the fluorescence intensity of 5-ALA-PpIX was higher than AME-PpIX peaking at $9 \mathrm{~h}$ post incubation. At $1 \mathrm{~h}$ post incubation the $\mathrm{T} / \mathrm{N}$ ratio of 5-ALA-PpIX was 4.37 times higher than AME-PpIX. AME-PpIX peaked at $6 \mathrm{~h}$ after which it declined whereas 5-ALA-PpIX continued to rise before it began its downward trend beyond $9 \mathrm{~h}$. In the case of exogenous PpIX and PME, it was found that PME exhibited higher $\mathrm{T} / \mathrm{N}$ fluorescence intensity ratio following the same pattern as that of 5-ALA-PpIX. It was found to be increasing up to $9 \mathrm{~h}$ post incubation where the $\mathrm{T} / \mathrm{N}$ ratio of PME was 6.61 times more than that of PpIX. By $24 \mathrm{~h}$ while 5-ALA-PpIX; AME-PpIX and exogenous PpIX almost reached base levels, the $\mathrm{T} / \mathrm{N}$ ratio of PME was still high, being almost 8.7 times higher than the $\mathrm{T} / \mathrm{N}$ ratio of PpIX.

To assess the efficacy of the topical application, it was compared with intravenous administration. Tumour bearing mice were divided into groups that received topical application or intravenous administration of 5-ALA, AME, PpIX or PME. Fig. 2 shows the fluorescence intensity of tumour xenografts compared with surrounding normal tissue when i.v. 5-ALA or AME was administered. The 5-ALA-PpIX and AME-PpIX fluorescence was highest in the first hour post administration. Though the overall fluorescence was high, the $\mathrm{T} / \mathrm{N}$ differential was also found to be the largest and significant at $1 \mathrm{~h}$ post 5 -ALA administration $(\mathrm{p}<0.05)$. At later time-points the differential was not significantly large and overall fluorescence decreased drastically by $6 \mathrm{~h}$. AMEPpIX fluorescence on the other hand, was found to be lower than that of the parent compound and the differential was not significant at any time-point. Intravenous administration of exogenous PpIX and PME did not produce macroscopic 


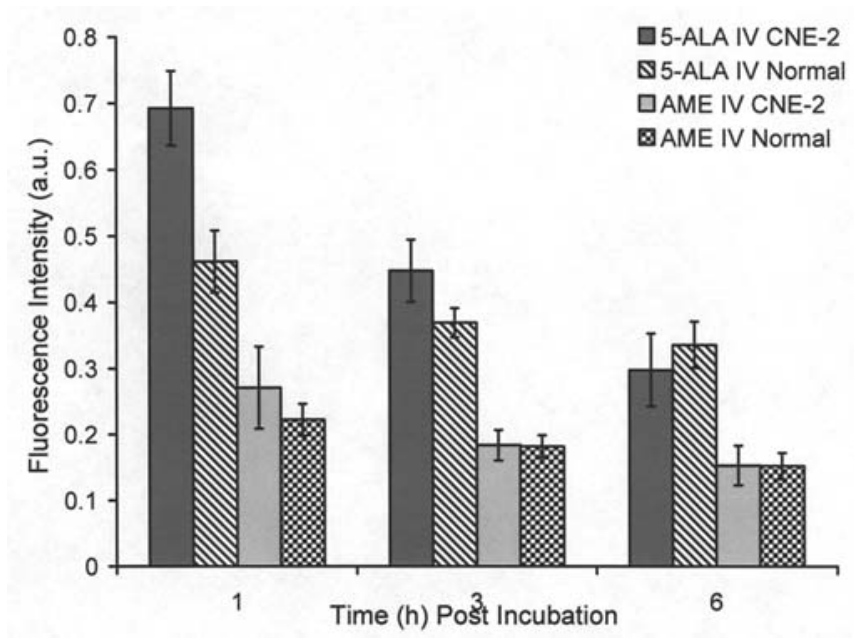

Figure 2. Fluorescence intensity of tumour and surrounding normal tissue when 5-ALA or AME was administered intravenously to tumour bearing mice.

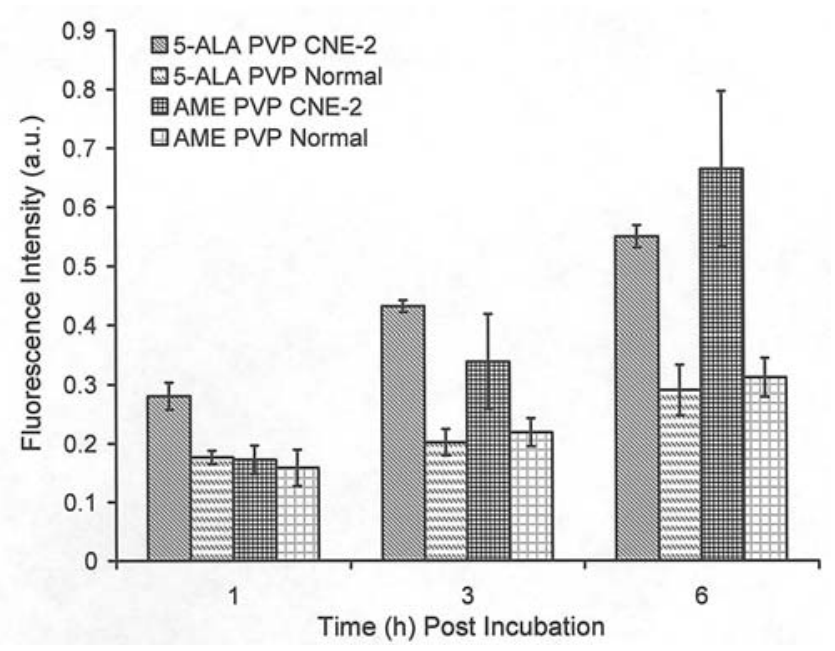

Figure 3. Fluorescence intensity of tumour xenografts compared with surrounding normal tissue post 5-ALA/AME topical applications on tumour bearing mice.

fluorescence as expected. Therefore no fluorescence images were obtained to compare.

When 5-ALA and AME topical applications were administered to the tumour bearing mice, the fluorescence pattern observed was opposite to that of the i.v. administration. The tumour fluorescence intensity post 5-ALA and AME topical application gradually increased. By $3 \mathrm{~h}$, there was significant difference between tumour and normal for both 5-ALA and AME topical applications (Fig 3). By $6 \mathrm{~h}$ the fluorescence intensity of the tumour xenografts was 2 times higher than that of normal surrounding tissue $(\mathrm{p}<0.05)$. Both 5-ALA and AME produced comparable PpIX fluorescence. Fluorescence was not observed in distal tissue.

In the case of PpIX and PME, three different types of gels were used. Gel 1 (8:2 PVP:Gantrez); Gel 2 (Gantrez with Stabilez 06) and Gel 3 (6:4 PVP:Gantrez). PME in Gel 1, which has higher content of PVP, gave the best results in terms of $\mathrm{T} / \mathrm{N}$ ratio (Fig. 4), 1.37, 1.57 and 1.88 at 1,3 and $6 \mathrm{~h}$

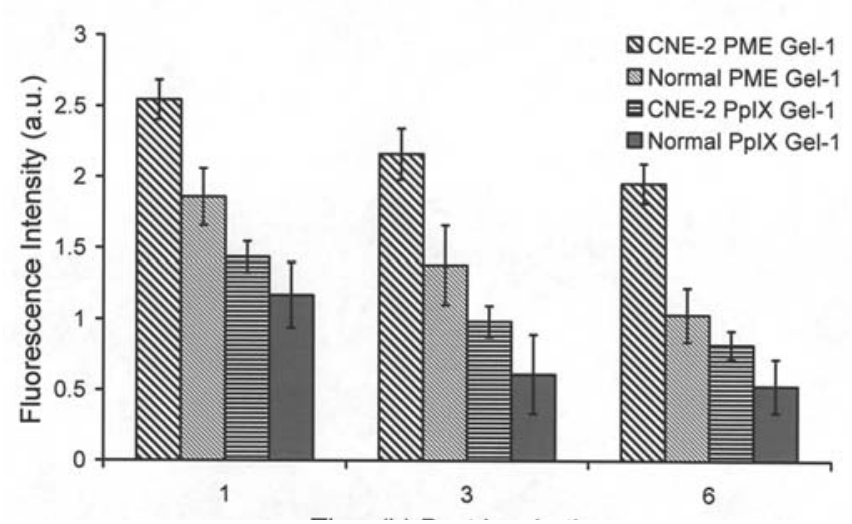

Figure 4. Fluorescence intensity of PpIX and PME topical application on tumour and surrounding normal.

post application respectively ( $\mathrm{p}<0.05$ at all time-points). PpIX in Gel 1 gave significant tumour to normal differential at 3 and $6 \mathrm{~h}$. The overall fluorescence was high in the case of PME for all gels. The other combinations with PpIX and PME did not have significant $\mathrm{T} / \mathrm{N}$ ratio or tumour selectivity and therefore are not discussed here.

\section{Discussion}

In the last decade, much effort has been made in developing good imaging systems and photosensitizers for fluorescence diagnosis. During this time, porphyrin based fluorescence diagnosis of superficial cancers in bladder, cervix, esophagus and brain has been widely investigated $(1,3,4,16-18)$. FDA approved synthetic photosensitizer such as Photofrin was in the center stage for a long time until second generation photosensitizers such as 5-ALA, ALA esters, hypericin and chlorins were introduced. Clinical trials using 5-ALA in the detection of oral and bladder cancer in our centre has been documented $(3,4,17)$. In these trials, 5-ALA was administered as an oral rinse or bladder instillation.

This study was carried out to investigate the uptake and retention pharmacokinetics of 5-ALA and AME in terms of endogenous PpIX production in human nasopharyngeal carcinoma NPC/CNE-2 cells in both in vitro and in vivo conditions. This was then compared to the effects of administration of exogenous PpIX and its ester derivative PME. Subsequently this tumour cell line was xenografted on the flanks of Balb/c nude mice to study the uptake and retention kinetics in an in vivo system. There have been several studies using ester derivatives of 5-ALA (19-22) namely hexyl-ester for PDT and PDD based on the rationale that esters are transported into cells at a faster rate. Up to date no extensive studies have been conducted using exogenous PpIX and its ester derivative PME in PDT or PDD of cancer cells. Endogenous PpIX is produced in the mitochondria and transported to the cytoplasm. Exogenous PpIX and PME enter the cytoplasm and target the mitochondria (23). Intravenous 5-ALA/AME administration not only gives high fluorescence of tumour but also that of normal distal organs such as the skin, liver and intestines at 1 $\mathrm{h}$ post administration. This can be attributed to considerable 
amounts of circulating 5-ALA/AME inducing local PpIX production. AME, in this study, did not induce as much PpIX as its long-chained counter parts such as octyl, hexyl and butyl esters (24). For both the in vitro and in vivo parts (intravenous administration) of the study, it was observed to induce less PpIX than 5-ALA. Prior studies have suggested that PpIX accumulation is dependent on the mode of application of 5-ALA, the microenvironment of the cells and other specific characteristics of the cell such as the size, ferrochelatase activity, cell cycle or cell density (25-27). This also means that it is tumour specific. The same observation was made in another study conducted by us using bladder carcinoma cells where AME induced more PpIX in vitro and in vivo than 5-ALA did in the same cells (11).

On the contrary, intravenous PpIX and PME did not exhibit fluorescence in the tumour or the surrounding normal tissue. Therefore, no comparison could be made between the drugs. Topical applications were compared in this case. Topical application of AME induced comparable PpIX fluorescence when compared with 5-ALA as opposed to the in vitro studies and i.v. administration. The topical application of AME allows for the longer retention of drug, which is bound to the cell membrane. When adjacent skin was analysed for PpIX fluorescence, it was found that the AME induced less cutaneous sensitivity when compared to 5-ALA.

Thus, there are merits for 5-ALA and AME delivery via topical applications. This delivery system maybe in many forms such as rinses, gels or creams, which have to be constituted extemporaneously when needed due to instability of 5-ALA. Films prepared for this study can be made and stored at room temperature for three to six months. These topical applications also have muco-adhesive properties and can be good candidates for use in the oral cavity PDD and PDT. Oral rinses for the mouth require patient to continuously rinse for $20 \mathrm{~min}$. Hence oral rinses are not practical for routine clinical use. Also the contact time for drug on oral tissue is short with the use of an oral rinse.

No significant differences were observed in the in vivo performance between 5-ALA and AME applications. However, this seems to be tumour dependent (11). In the case of PpIX and PME topical gel application, esterification seems to reduce the rate of photosensitizer degradation. The difference also lies in the gel composition. Gels with high content of PVP seem to exhibit high selectivity. The drugs were dissolved in different hydrophilic gel composites. These gels contained Gantrez and PVP in different proportions or Gantrez and Stabileze 06. Gel-1 and Gel-3 have the same composition differing in ratios of Gantrez and PVP. Nevertheless they both are similar to oil-in-water emulsion where Gantrez may be considered as the oil component and the PVP as the water component. Oil-in-water emulsions are known to enhance lipophilic ALA hexylester localization in tumour whereas saline-DMSO-ethanol composition enhances amphiphilic 5-ALA (zwitterions) localization (28). In this study it was observed that PME in Gel-1 exhibited statistically significant selectivity at all time-points. Since PME is lipophilic, the oil-in-water emulsion resulted in significant selectivity from $1 \mathrm{~h}$ post application. On the other hand, PpIX being amphiphilic may have worked better if it were constituted in saline-DMSO-ethanol mixture.
In conclusion, 5-ALA induced more PpIX than AME in NPC/CNE-2 cells in vitro. Intravenous administration of 5-ALA only had significant selectivity at the earliest time-point of $1 \mathrm{~h}$ after which there was no significant $\mathrm{T} / \mathrm{N}$ differential whereas AME induced low PpIX fluorescence and no significant $\mathrm{T} / \mathrm{N}$ differential. Exogenous PpIX and PME show promise as photosensitizers as they exhibit significant selectivity between tumour and normal tissue. Modification of delivery vehicle used for application of exogenous PpIX and PME could allow for rapid and even distribution of photosensitizer and clearance from normal tissue.

\section{Acknowledgements}

The authors would like to thank National Cancer Centre of Singapore (NCCS) and National Medical Research Council (NMRC) for supporting this work.

\section{References}

1. Baumgartner R, Huber RM, Schulz H, Stepp H, Rick K, Gamarra F, Leberig A and Roth C: Inhalation of 5aminolevulinic acid: a new technique for fluorescence detection of early stage lung cancer. J Photochem Photobiol B 36: 169$174,1996$.

2. Kennedy JC and Pottier RH: Endogenous protoporphyrin IX, a clinically useful photosensitizer for photodynamic therapy. J Photochem Photobiol B 14: 275-292, 1992.

3. Zheng W, Soo KC, Sivanandan R and Olivo M: Detection of neoplasms in the oral cavity by digitized endoscopic imaging of 5-aminolevulinic acid-induced protoporphyrin IX fluorescence. Int J Oncol 21: 763-768, 2002.

4. Zheng W, Soo KC, Sivanandan R and Olivo M: Detection of squamous cell carcinomas and pre-cancerous lesions in the oral cavity by quantification of 5-aminolevulinic acid induced fluorescence endoscopic images. Lasers Surg Med 31: 151-157, 2002.

5. Tammilmani V, Yee KK, Heng PW, Soo KC and Olivo M: An evaluation of exogenous application of protoporphyrin IX and its dimethyl ester as a photodynamic diagnostic agent in poorly differentiated human nasopharyngeal carcinoma. Photochem Photobiol 80: 595-601, 2004.

6. Moan J, Christensen T and Jacobsen PB: Porphyrin localization and treatment of tumours. In: Progress in Clinical and Biological Research. Gomer CJ (ed). Alan R. Liss, Inc., New York, pp413-442, 1984.

7. Kepczynski M, Pandian RP, Smith KM and Ehrenberg B: Do liposome-binding constants of porphyrins correlate with their measured and predicted partitioning between octanol and water? Photochem Photobiol 76: 127-134, 2002.

8. Berenbaum MC, Bonnett R and Scourides PA: In vivo biological activity of the components of haematoporphyrin derivative. Br J Cancer 45: 571-581, 1982.

9. Scott JJ: The biosynthesis of porphyrins and porphyrin metabolism. In: Ciba Foundation symposium. Churchill, London, p43, 1955.

10. Manivasager V, Heng PW, Hao J, Zheng W, Soo KC and Olivo M: Macro-microscopic fluorescence imaging of human NPC xenografts in a murine model using topical vs intravenous administration of 5-aminolevulinic acid. Int $\mathbf{J}$ Oncol 21: 1003-1007, 2002.

11. Manivasager V, Heng PW, Hao J, Zheng W, Soo KC and Olivo M: A study of 5-aminolevulinic acid and its methyl ester used in in vitro and in vivo systems of human bladder cancer. Int J Oncol 22: 313-318, 2003.

12. Duffner F, Ritz R, Freudenstein D, Weller M, Dietz K and Wessels J: Specific intensity imaging for glioblastoma and neural cell cultures with 5-aminolevulinic acid-derived protoporphyrin IX. J Neurooncol 71: 107-111, 2005.

13. Van Hillegersberg R, van den Berg JW, Kort WJ, Terpstra OT and Wilson JH: Selective accumulation of endogenously produced porphyrins in a liver metastasis model in rats. Gastroenterology 103: 647-651, 1992. 
14. Whitaker CJ, Battah SH, Forsyth MJ, Edwards C, Boyle RW and Matthews EK: Photosensitization of pancreatic tumour cells by delta-aminolaevulinic acid esters. Anticancer Drug Des 15: 161-170, 2000.

15. Navone NM, Polo CF, Frisardi AL, Andrade NE and Battle AM: Heme biosynthesis in human breast cancer - mimetic 'in vitro' studies and some heme enzymic activity levels. Int J Biochem 22: 1407-1411, 1990.

16. Cheng CW, Lau WK, Tan PH and Olivo M: Cystoscopic diagnosis of bladder cancer by intravesical instillation of 5aminolevulinic acid induced porphyrin fluorescence - the Singapore experience. Ann Acad Med Singapore 29: 153-158, 2000.

17. Olivo M, Lau WK, Manivasager V, Tan PH and Cheng CW: Fluorescence confocal microscopy and image analysis of bladder cancer using 5-aminolevulinic acid. Int $\mathrm{J}$ Oncol 22: 523-528, 2003.

18. Friesen SA, Hjortland GO, Madsen SJ, Hirschberg H, Engebraten O, Nesland JM and Peng Q: 5-Aminolevulinic acidbased photodynamic detection and therapy of brain tumors (review). Int J Oncol 21: 577-582, 2002.

19. Zenzen V and Zankl H: Protoporphyrin IX-accumulation in human tumor cells following topical ALA- and h-ALAapplication in vivo. Cancer Lett: 35-42, 2003.

20. Lange N, Jichlinski P, Zellweger M, Forrer M, Marti A, Guillou L, Kucera P, Wagnieres G and van den Bergh H: Photodetection of early human bladder cancer based on the fluorescence of 5-aminolaevulinic acid hexylester-induced protoporphyrin IX: a pilot study. Br J Cancer 80: 185-193, 1999.

21. Endlicher E, Gelbmann CM, Knuchel R, Furst A, Szeimies RM, Golder SK, Scholmerich J, Lottner C and Messmann H: Hexaminolevulinate-induced fluorescence endoscopy in patients with rectal adenoma and cancer: a pilot study. Gastrointest Endosc 60: 449-454, 2004.
22. Wu SM, Ren QG, Zhou MO, Wei Y and Chen JY: Photodynamic effects of 5-aminolevulinic acid and its hexylester on several cell lines. Sheng Wu Hua Xue Yu Sheng Wu Wu Li Xue Bao (Shanghai) 35: 655-660, 2003.

23. Yee KK, Soo KC, Bay BH and Olivo M: A comparison of protoporphyrin IX and protoporphyrin IX dimethyl ester as a photosensitizer in poorly differentiated human nasopharyngeal carcinoma cells. Photochem Photobiol 76: 678-682, 2002.

24. De Rosa FS, Lopez RF, Thomazine JA, Tedesco AC, Lange N and Bentley MV: In vitro metabolism of 5-ALA esters derivatives in hairless mice skin homogenate and in vivo PpIX accumulation studies. Pharm Res 21: 2247-2252, 2004.

25. Wyld L, Smith O, Lawry J, Reed MW and Brown NJ: Cell cycle phase influences tumour cell sensitivity to aminolaevulinic acidinduced photodynamic therapy in vitro. Br J Cancer 78: 50-55, 1998.

26. Wyld L, Reed MW and Brown NJ: The influence of hypoxia and $\mathrm{pH}$ on aminolaevulinic acid-induced photodynamic therapy in bladder cancer cells in vitro. Br J Cancer 77: 1621-1627, 1998.

27. Moan J, Bech O, Gaullier JM, Stokke T, Steen HB, Ma LW and Berg K: Protoporphyrin IX accumulation in cells treated with 5-aminolevulinic acid: dependence on cell density, cell size and cell cycle. Int J Cancer 75: 134-139, 1998.

28. Casas A, Perotti C, Fukuda H, Rogers L, Butler AR and Batlle A: ALA and ALA hexyl ester-induced porphyrin synthesis in chemically induced skin tumours: the role of different vehicles on improving photosensitization. Br J Cancer 85: 1794-1800, 2001. 